\title{
Correlation of Six Minute Walk Test and Lung Function Test Variables (\% FEV1, \% FVC, \% DLCO) in Patients with Connective Tissue Disorder - Interstitial Lung Disease
}

\author{
Seema S1, Nalini Jayanthi Nagesh², Subramanian Suriyan³ ${ }^{3}$ Ram Phanindra Talatam ${ }^{4}$ \\ ${ }^{1}$ Department of Respiratory Medicine, SRM Medical College Hospital \& Research Centre, Chennai, \\ Tamilnadu, India. ${ }^{2}$ Department of Respiratory Medicine, SRM Medical College Hospital \& Research \\ Centre, Chennai, Tamilnadu, India. ${ }^{3}$ Department of Respiratory Medicine, SRM Medical College \\ Hospital \& Research Centre, Chennai, Tamilnadu, India. ${ }^{4}$ Department of Respiratory Medicine, SRM \\ Medical College Hospital \& Research Centre, Chennai, Tamilnadu, India.
}

ABSTRACT

\section{BACKGROUND}

Interstitial Lung Diseases (ILDs) also known as diffuse parenchymal lung disease, include a group of diffuse parenchymal infiltrative lung diseases. A restrictive defect is the most frequent pulmonary abnormality in patients with pulmonary fibrosis which is the usual consequence of many ILDs. Connective tissue disorders are usually rare, but are potentially life threatening conditions. The spectrum of ILD varies from mucocutaneous symptoms, arthralgia / arthritis to impairment of pulmonary and renal function. Systemic Lupus Erythematosus (SLE), Systemic Sclerosis (SSc), Sjogren's Syndrome (SS), inflammatory muscle diseases and overlap-syndromes are grouped together as connective-tissue disorders. Involvement of lung and its function is the most common form of interstitial lung disease, leading to high morbidity and mortality among the group of connective tissue disorders. We wanted to correlate the 6-Min Walk Distance (6MWTD) with the Pulmonary Function Test values such as \% FEV1, \% FVC and FEV1 / FVC, among patients with connective tissue disorder associated Interstitial Lung Disease in SRM Medical College Hospital, a tertiary care hospital in Tamilnadu, South India.

\section{METHODS}

This study was done as a cross sectional analytical study among 31 patients in the Department of Respiratory Medicine, Rheumatology and General Medicine in SRM medical college and hospital. Each patient was explained the purpose of the study and the need for complete co-operation. Those who satisfied the inclusion and exclusion criteria were included. Data was collected using a structured proforma. 6minute walk test and pulmonary function tests were performed and correlated.

\section{RESULTS}

There appears to be a significant correlation between 6-minute walk test, \% desaturation, 6 Minute Walk Test Distance, 6 Minute Walk Test Pre BDI and 6 Minute Walk Test Post BDI with spirometry values especially with the Spirometry FEV $1 \%$, Spirometry FVC \% and \% predicted DLCO.

\section{CONCLUSIONS}

6MWT can be used as a useful surrogate for pulmonary function tests especially among the population where spirometry is not possible. 6MWT has good reproducibility, is simple to perform and can be used as a screening tool for Interstitial Lung Disease in connective tissue disorder patients.

\section{KEY WORDS}

Six Minute Walk Test, 6-MWT, Pulmonary Function Test, Lung Function Test, Connective Tissue Disorder, Interstitial Lung Disease
Corresponding Author:

Dr. N. Nalini Jayanthi.

35, Udaya Nagar Main Road,

Porur-600116, Chennai, India.

E-mail: nalinijayanthi59@gmail.com

DOI: $10.14260 / j e m d s / 2020 / 585$

How to Cite This Article:

Seema S, Nagesh NJ, Suriyan $S$, et al. Correlation of six minute walk test and lung function test variables (\% fev1, \%fvc, $\%$ dlco) in patients with connective tissue disorder - interstitial lung disease. J Evolution Med Dent Sci 2020;9(37):26902694, DOI: 10.14260/jemds/2020/585

Submission 26-04-2020,

Peer Review 03-08-2020,

Acceptance 10-08-2020,

Published 14-09-2020.

Copyright (C) 2020 JEMDS. This is an open access article distributed under Creative Commons Attribution License [Attribution 4.0 International (CC BY 4.0)] 


\section{BACKGROUND}

Interstitial Lung Diseases (ILDs) also known as diffuse parenchymal lung diseases, include a group of diffuse parenchymal infiltrative lung diseases which are further classified according to etiologic, clinical, radiologic, and histopathologic features. The most common form of the ILD is Idiopathic Pulmonary Fibrosis (unknown origin). 1-3 Fibrosis of the alveolar interstitium is more common, chronic alveolitis (the central mechanism and distinguishing feature) produces a functional derangement leading to loss of functional gas exchange units leading to "end stage lung". 4

Histological diagnosis by biopsy remains the diagnostic "gold standard" for ILD. A restrictive defect is the most frequent pulmonary abnormality in patients with pulmonary fibrosis which is the usual consequence of many ILDs. Restrictive defect is a typical clinical presentation in patients with IPF. Instituting an accurate diagnosis of ILD can be challenging for clinicians since there are more than two hundred different subtypes. ${ }^{5}$

Connective tissue disorders are usually rare, but a potentially life threatening condition. Connective tissue disorders affect people of all ages, having higher preponderance for young adults especially women. The spectrum of the disease varies from mucocutaneous symptoms, arthralgia/arthritis to impairment of Pulmonary and renal function. ${ }^{6}$ Systemic lupus erythematosus (SLE), systemic sclerosis (SSc), Sjogren's syndrome (SS), inflammatory muscle diseases and overlap-syndromes are grouped together as connective-tissue disorders. ${ }^{7}$

SLE is a prototypic autoimmune disease and it is one of the most common autoimmune disorders in women of reproductive age group. $6,8 \mathrm{SS}$ is a slow progressive disease, of inflammatory autoimmune aetiology mainly affecting principally the exocrine glands. SSc, also called as scleroderma, is a multisystem disorder. SSc is characterized by structural and functional irregularities of small blood vessels, fibrosis of the skin and internal organs, activation of the immune system and autoimmunity. ${ }^{9}$

Polymyositis and dermatomyositis comprise of weakness of symmetric muscle, myositis, elevated levels of serum skeletal muscle enzymes, typical electromyography pattern and, in case of dermatomyositis, presenting with typical rash. ${ }^{10}$ Overlap syndromes are characterised by a combination of one or more rheumatic disease symptoms in the same patient. Raynaud's phenomenon, arthritis and sclerodactyly are most commonly presenting features. When they present with Polymyositis and fibrosing alveolitis they are more serious. ${ }^{6}$

Involvement of Lung and its function in connective tissue diseases is usually related with considerable morbidity rate and death. This is especially most common in the form of interstitial lung disease, which can happen in any of these individual disorders among the group of connective tissue disorders. ${ }^{11}$ The 5 -year mortality rate is three times more in patients with rheumatoid arthritis (RA) and scleroderma who is suffering from associated ILD. ${ }^{12,13}$ However, when compared to those patients with idiopathic interstitial pneumonias (IIPs), patients with CTD - ILD responds well with the immunosuppressive therapy and have an enhanced prognosis pattern. ${ }^{14}$
The routine baseline and follow up work up for the patients with ILD involves Chest X-ray (non-specific infiltrates), HRCT scan (nodules, cysts, ground glass change, honeycomb change, traction bronchiectasis, intra-lobular septal thickening), Pulse oximetry/ arterial blood gas (Low Sp02, Low PaO2), Connective tissue disease serology (positive for auto antibodies e.g. ANA, ENA, RF, myositis antibodies, ANCA), Lung function tests (spirometry, lung volumes, DLCO), Low FEV1, FVC, Normal or high FEV1 / FVC ratio, Reduced lung volumes, Reduced DLCO and 6-minute walk test (reduced walk distance, oxygen desaturation). ${ }^{15}$

There is a paucity of literature on the pattern, determinants, distribution and response of treatment of ILD in India. Small regional studies have contributed a lot in our understanding of the disease, though no true epidemiological study on prevalence of ILDs and its different subgroups from India is available. This study aims to study the clinical and radiological profile of patients with connective tissue disorder presenting with interstitial lung disease This study attempts to evaluate the associated risk factors with the pattern of the disease using PFT ( \% FEV1, \% FVC, FEV1 / FVC \& \% DLCO in spirometry), 6-min walk distance (6MWTD), oxygen desaturation $(\Delta \mathrm{SO} 2)$ and arterial blood gases in ILD patients.

\section{Objectives}

This study aims to correlate the 6-min walk distance (6MWTD) with the Pulmonary Function Test values such as \% FEV1, \% FVC and FEV1 / FVC, among Interstitial Lung Diseases in connective tissue disorder patients of SRM Medical College, a tertiary care hospital in Tamil Nadu, South India.

\section{METHODS}

This study was done by a cross sectional analytical study design among 31 inpatients and outpatients, attending the Department of Respiratory Medicine, Rheumatology and General Medicine in SRM Medical College and Hospital, a tertiary care hospital in Tamilnadu, South India. According to Muhammed Shafeeq K et al study, the prevalence of Idiopathic Pulmonary Fibrosis (commonest form of ILD) as $38.6 \%{ }^{16}$ With a precision of $17.1 \%$ and $95 \%$ confidence interval, the sample size is calculated as

$\mathrm{N}=\frac{\mathrm{Z} 21-\alpha / 2 * \mathrm{p} *(1-\mathrm{p})}{\mathrm{d}^{2}}$

Where,

Z1- $\alpha$ / 2 - two tailed probability for $95 \%$ confidence interval = 1.96

$\mathrm{p}(\%)$ - prevalence of $=0.386$

$\mathrm{d}(\%)$ - precision or allowable error for $=0.175$

$N=\frac{1.96^{\wedge} 2 * 0.386 *(1-0.386)}{0.175^{\wedge} 2}$

$\mathrm{N}=29.73$

Thus, the total sample size required for the study is 30 . All patients irrelevant of gender, between the age of 18-80 years with symptoms suggestive of ILD and CTD who were willing to 
participate were included in the study. Patients with Respiratory illnesses other than ILD, Occupational lung disorders, Chronic Cardiac, Hepatic and Renal disease were excluded from the study. The minimum calculated sample size is 30 . However, in the study period of 18 months, 31 subjects were included in the study.

\section{Procedure}

Institutional Ethical Committee approval was taken prior to the study. Consecutive sampling was followed. After establishing rapport with the study subject, the purpose, procedure, benefits, risks and confidentiality of the study were explained. Informed written consent from the study subject was taken before the interview schedule was administered. Data was collected from the eligible patients on basic Patient's history was taken according to the questionnaire (with personal, environmental, occupational and clinical details) and subjected to clinical examination.

Interstitial lung disease was diagnosed by the following methods. 17

- History and physical examination.

- Autoantibodies for connective tissue disorders.

- $\quad$ Spirometry - PFT and DLCO.

- 6-minute walk test.

\section{Statistical Analysis}

The data was coded and entered in Microsoft Excel and analysed using SPSS version 20. Frequencies and percentages with visualisation was used for categorical variables such as gender, type of illness. Measures of central tendency and dispersion were used for numerical variables from the serum blood levels such as age, 6-MWT and PFT parameters. Pearson's Correlation test was used to predict the association between 6-MWT and PFT parameters. P-value of less than 0.05 was considered as statistically significant.

\section{RESULTS}

The study population comprised of 31 Interstitial Lung Disease patients. The mean and median age of the study population is 54 and 55 respectively. Majority $(13,42 \%)$ of the study population were belonging to 51-60 age group category. 18 (58 \%) were females and 13 (42\%) were males. 8 (26\%) were history of smoking. 5 (16\%) were history of alcoholism. The basic socio-demographic details of the study subjects are represented in table 1.

In the study population, the mean Duration of illness in months is 7.19 with Std. Deviation of 2.548. The median and mode of the duration of illness is six. All of them presented with cough and shortness of breath. Two (6.5\%) of the study population had presented with haemoptysis also. Majority $(12$, $39 \%$ ) of the study population presented with the joint pain and swelling. This is followed by skin rashes, skin thickening, Raynaud's phenomenon and difficulty in swallowing. Most common presenting symptom is pitting scars $(5,16.1 \%)$ followed by pallor and clubbing. All the participants were having positive CRP status. ANA is 1+ among 13 (42\%) and 2+ among 11 (36\%). Table 2 represents the clinical details of the study subjects.

\begin{tabular}{|c|c|c|c|c|}
\hline Sl. No. & Variable & Category & Frequency & Percent \\
\hline \multirow{5}{*}{1} & \multirow{5}{*}{ Age } & $<30$ & 1 & 3.2 \\
\hline & & $31-40$ & 1 & 3.2 \\
\hline & & $41-50$ & 9 & 29.0 \\
\hline & & $51-60$ & 13 & 41.9 \\
\hline & & $>60$ & 7 & 22.6 \\
\hline \multirow[b]{2}{*}{2} & \multirow[b]{2}{*}{ Gender } & Female & 18 & 58.1 \\
\hline & & Male & 13 & 41.9 \\
\hline \multirow{2}{*}{3} & \multirow{2}{*}{ Smoking } & Yes & 8 & 26 \\
\hline & & No & 23 & 74 \\
\hline \multirow{3}{*}{4} & \multirow{3}{*}{ Alcoholism } & Yes & 5 & 16 \\
\hline & & No & 26 & 84 \\
\hline & & Total & 31 & 100.0 \\
\hline
\end{tabular}

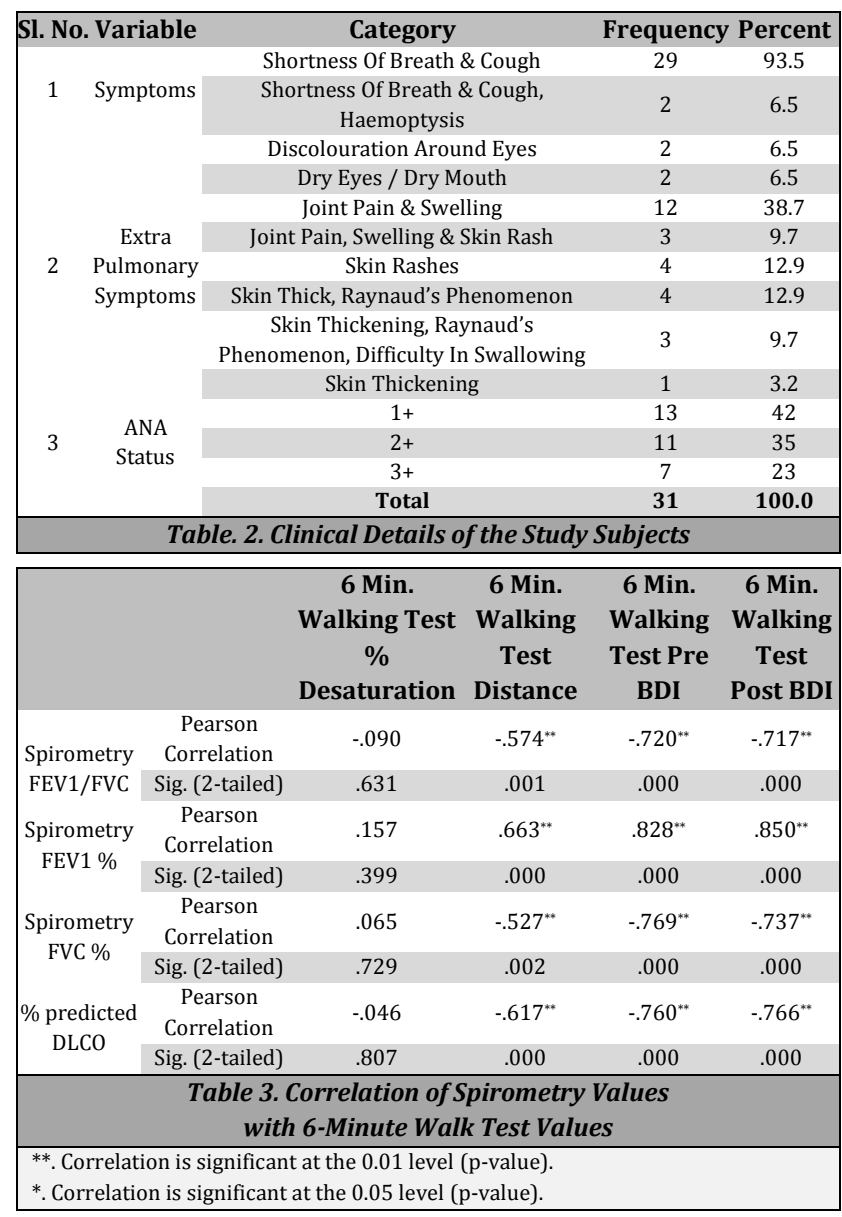

The mean six-minute walk distance of the study population was 352.26 with a standard deviation of 57.36. In Six- Minute Walk Test, the parameters such as 6-MWT SpO2 pre-test, 6MWT SpO2 post-test, 6-MWT SpO2 \% desaturation, 6-MWT Distance, 6-MWT pre BDI and 6-MWT post BDI. When 6-MWT and PFT parameters were subjected to correlation tests, 6 Minute Walking test Distance, 6 Minute Walking test Pre BDI and 6 Minute Walking test Post BDI were significantly correlated with Spirometry FEV1 / FVC, Spirometry FEV1 \%, Spirometry FVC \% and \% predicted DLCO with p-value of less than 0.05. Table 3. Represents the Correlation of Spirometry values with 6-Minute walk test values.

\section{DISCUSSION}

This study was conducted in a tertiary healthcare center in Chennai, Tamilnadu, India, to evaluate the relation between walk distance (6MWTD), and parameters of 6MWT with PFT 
(\% FEV1, \% FVC, FEV1 / FVC \& \% DLCO) in ILD in CTD patients. In this study, there appears to be significant correlation between 6 Minute walk test \% Desaturation, 6 Minute walk test Distance, 6 Minute walk test Pre BDI and 6 Minute walk test Post BDI with the spirometry values especially with the Spirometry FEV \%, Spirometry FVC \% and $\%$ predicted DLCO with a p-value less than 0.05 . They were mainly negative correlations, ie., The increase in one variable will cause decrease in the other variable.

Deuschle $\mathrm{K}$ et al, studied the usefulness of Six-minute walk distance test and its utility as a marker for disability and complaints in 101 consecutive patients with systemic sclerosis. The median 6MWDistance was $491.0 \mathrm{~m}$ (range 86.0$664.5 \mathrm{~m}$ ). 6-MWDistance weak-to-moderate correlation with predicted FVC, FEV1, TLC, DLCO and nutrition status of the patients. There was a weaker correlations for age and BMI.18 Lima TR et al, studied the relationship between functional capacity, mobility of the joints and lung function in patients with systemic sclerosis and concluded that 6 MWD can be influenced by cardiovascular and pulmonary impairments in systemic sclerosis, and further they suggested that the musculoskeletal dysfunction play an significant role in the functional capacity. ${ }^{19}$ 6MWT and its components may indicate patients at amplified possibility of developing pulmonary hypertension. 20

Villalba WO et al, studied the usefulness of Six-minute walk test for the evaluation of severity pulmonary function in 110 scleroderma patients. Three variables were statistically significant when tested with six-minute walk distance such as age, race, and dyspnoea index. ${ }^{21}$ Antonella Caminati et al, studied the Walking distance on 6-Minute Walk Talk test as a prognostic factor in idiopathic pulmonary fibrosis. Patients walking less than 212 metres had a significantly lesser survival rate than those walking Further. 6 min walk distance was independently associated with mortality. ${ }^{22}$

In a study by Sébastien Sanges et al, the 6-Min Walk Distance is independently related with initial Heart Rate and Heart Rate variation; with Pulmonary Arterial Hypertension but not Interstitial Lung Disease, signifying that pulmonary vasculature may have a superior influence than parenchymal connection on limitation of the function. ${ }^{23}$ Since Six Minute Walk Test is a submaximal exercise test, the diagnosis of chronotropic incompetence cannot be certainly made. The main limitation of this study is probable confounding variables such as Musculo skeletal pain levels and pulmonary artery pressure were not studied.

$6 \mathrm{MWT}$ is feasible and reliable test for the severity assessment in interstitial lung disease. ${ }^{24}$ In our study, the mean six-minute walk distance was 352.26 with a standard deviation of 57.36. Schoindre $\mathrm{Y}$ et al, concluded that there is lack of specificity of the 6-minute walk test as an outcome measure for their 87 patients with systemic sclerosis. Twentysix patients (30\%) out of 87 patients had an abnormal 6MWTest and the mean 6MW Distance was 461.8 + / - 103.0 metre. C-reactive protein (CRP) was the single independent variable related with abnormal 6MWTest. ${ }^{25}$ 6-Minute Walk Test can be used as useful surrogate for pulmonary function tests especially among the population where spirometry is not possible.

Future studies can be done with a larger sample so that inferences will be with higher precision, multi-centred so that generalisability to the whole population can be better and follow up and data on survival so that it will reveal the prognostic factors and factors related to survival can be studied.

\section{CONCLUSIONS}

When 6-MWT and PFT parameters were subjected to correlation tests, 6 Minute Walk Test Distance, 6 Minute Walk Test Pre BDI and 6 Minute Walk Test Post BDI were significantly negatively correlated with Spirometry FEV / FVC, Spirometry FEV1 \%, Spirometry FVC \% and \% predicted DLCO with a p-value of less than 0.05. 6MWT has good reproducibility, is simple to perform and can be used as a screening tool for Interstitial Lung Disease in Connective tissue disorder patients.

Written informed consent was obtained from each patient. Ethical clearance was obtained from Institutional Ethical Committee, SRM Medical College, Chennai.

We thank the institution for its constant support and encouragement We also thank the study subjects for their cooperation.

Financial or Other Competing Interests: None.

\section{REFERENCES}

[1] Fischer A, du Bois R. Interstitial lung disease in connective tissue disorders. Lancet 2012;380(9842):689-98.

[2] Fischer A, West SG, Swigris JJ, et al. Connective tissue disease-associated interstitial lung disease: a call for clarification. Chest 2010;138(2):251-6.

[3] Vij R, Strek ME. Diagnosis and treatment of connective tissue disease-associated interstitial lung disease. Chest 2013;143(3):814-24.

[4] Crystal RG, Gadek JE, Ferrans VJ, et al. Interstitial lung disease: current concepts of pathogenesis, staging and therapy. Am J Med 1981;70(3):542-68.

[5] Wells AU, Hirani N, Bradley B, et al. Interstitial lung disease guideline: the British Thoracic Society in collaboration with the Thoracic Society of Australia and New Zealand and the Irish Thoracic Society. Thorax 2008;63 Suppl 5:v1-58.

[6] Gaubitz M. Epidemiology of connective tissue disorders. Rheumatology (Oxford) 2006;45 Suppl 3:iii3-4.

[7] White PH, Chang RW. Public health and arthritis: a growing imperative. In: Klippel JH, Stone JH, Crofford LJ, et al, eds. Primer on the rheumatic diseases. $13^{\text {th }}$ edn. New York, NY: Springer New York 2008:1-5.

[8] McCarty DJ, Manzi S, Medsger TA, et al. Incidence of systemic lupus erythematosus. Race and gender differences. Arthritis Rheum 1995;38(9):1260-70.

[9] Drosos AA, Andonopoulos AP, Costopoulos JS, et al. Prevalence of primary sjögren's syndrome in an elderly population. Br J Rheumatol 1988;27(2):123-7.

[10] Oddis CV, Conte CG, Steen VD, et al. Incidence of polymyositis-dermatomyositis: a 20-year study of hospital diagnosed cases in Allegheny County, PA 19631982. J Rheumatol 1990;17(10):1329-34. 
[11] Mira-Avendano I, Abril A, Burger CD, et al. Interstitial lung disease and other pulmonary manifestations in connective tissue diseases. Mayo Clin Proc 2019;94(2):309-25.

[12] Zamora-Legoff JA, Krause ML, Crowson CS, et al. Risk of serious infection in patients with rheumatoid arthritisassociated interstitial lung disease. Clin Rheumatol 2016;35(10):2585-9.

[13] Marigliano B, Soriano A, Margiotta D, et al. Lung involvement in connective tissue diseases: a comprehensive review and a focus on rheumatoid arthritis. Autoimmun Rev 2013;12(11):1076-84.

[14] Park JH, Kim DS, Park IN, et al. Prognosis of fibrotic interstitial pneumonia. Am J Respir Crit Care Med 2007;175(7):705-11.

[15] Tiwari A, Kumar K, Bhushan B, et al. Study of clinico radiological profile and treatment modalities in interstitial lung disease. Sch J Appl Med Sci 2016;4(3F):1086-105.

[16] Shafeeq MK, Nair S, Mathew M, et al. Aetiology and clinicradiological profile of interstitial lung disease in a tertiary care centre. Pulmon 2011;13(1):12-5.

[17] Vij R, Strek ME. Diagnosis and treatment of connective tissue disease-associated interstitial lung disease. Chest 2013;143(3):814-24.

[18] Deuschle K, Weinert K, Becker MO, et al. Six-minute walk distance as a marker for disability and complaints in patients with systemic sclerosis. Clin Exp Rheumatol 2011;29(2 Suppl 65):S53-9.
[19] Lima TRL, Guimarães FS, Silva LA, et al. Relationship between functional capacity, joint mobility and pulmonary function in patients with systemic sclerosis. J Bodyw Mov Ther 2015;19(1):17-24.

[20] Rizzi M, Sarzi-Puttini P, Airoldi A, et al. Performance capacity evaluated using the 6-minute walk test: 5 -year results in patients with diffuse systemic sclerosis and initial interstitial lung disease. Clin Exp Rheumatol 2015;33(4 Suppl 91):S142-7.

[21] Villalba WO, Sampaio-Barros PD, Pereira MC, et al. Sixminute walk test for the evaluation of pulmonary disease severity in scleroderma patients. Chest 2007;131(1):21722.

[22] Caminati A, Bianchi A, Cassandro R, et al. Walking distance on 6-MWT is a prognostic factor in idiopathic pulmonary fibrosis. Respir Med 2009;103(1):117-23.

[23] Sanges S, Giovannelli J, Sobanski V, et al. Factors associated with the 6-minute walk distance in patients with systemic sclerosis. Arthritis Res Ther 2017;19(1):279.

[24] Wilsher M, Good N, Hopkins R, et al. The six-minute walk test using forehead oximetry is reliable in the assessment of scleroderma lung disease. Respirology 2012;17(4):647-52.

[25] Schoindre Y, Meune C, Dinh-Xuan AT, et al. Lack of specificity of the 6-minute walk test as an outcome measure for patients with systemic sclerosis. J Rheumatol 2009;36(7):1481-5. 